\title{
Selection of herbaceous plant assortment for park ground cover using plants of natural phytocoenosis
}

\author{
Svetlana N. Shlapakova ${ }^{3,}{ }^{,}$, Irina V. Beriozkina ${ }^{1}$, Olga E. Hanbabayeva ${ }^{1}$, Vladimir N. Sorokopudov ${ }^{2}$, and Yevgeniy S. \\ Lukashov $^{3}$ \\ ${ }^{1}$ Russian State Agricultural University - Moscow Timiryazev Agricultural Academy, 127550 Moscow, Russia \\ ${ }^{2}$ All-Russian Horticultural Institute for Plant-Breeding, Agrotechnology and Nursery, 115598 Moscow, Russia \\ ${ }^{3}$ Bryansk State Engineering Technological University, 241037 Bryansk, Russia
}

\begin{abstract}
The modern direction of landscape architecture to creation of ecological and naturalistic plant communities of perennial herbaceous species for the design of park walking routes in the style of "naturgarten" is highlighted in this paper. The principles of selection of herbaceous perennials developed by Russian scientists for planting in urban environments, as well as the principles of creating combinatorial plantings are given. The range of perennial herbaceous plants proposed to create a ground cover along the park walking route with an indication of their environmental requirements is presented in the Tables. The type and storey of plantings where these plants can be used, the flowering period and in the note the additional features of a particular species are indicated. This material can be further used in the design of structural modules of the ground cover according to the type of natural phytocoenoses for park areas with different environmental conditions.
\end{abstract}

\section{Introduction}

There is a tendency to create ecological gardens and parks in which biodiversity is maintained and expanded. A sustainable landscape is created in an urbanized environment and the use of water, energy resources and labor costs for agrotechnical care of such plantations are rationalized. All this is clearly seen at the present stage of development of the world landscape architecture.

In this regard, the study of decorative and ecological features of plants of the natural phytocoenosis is relevant at the present stage.

\section{Research purpose}

The substantiation of the range of herbaceous plants to create a park ground cover on the basis of the plants of the natural phytocoenosis.

\section{Research tasks}

1. Study of ecological requirements (relation to illumination, humidity, $\mathrm{pH}$ and soil fertility) of the plants of the natural phytocoenosis.

2. Evaluation of the flowering period and other decorative features of the plants of the natural phytocoenosis.
Scientific novelty lies in the analysis of ecological requirements and ornamental qualities of the plants of the natural phytocoenosis to justify the range of herbaceous plants with the aim of designing green ground cover in the style of Naturgarten preserving the decorativeness during the whole season.

\section{Results}

The study of ecological requirements and decorative features of the plants of the natural phytocoenosis allows to design a ground cover for open, closed and semi-open park spaces. As a result, the artistic value of the spaces adjacent to the walking route significantly increases.

Historically, in Russia, in urban landscaping, the flower beds were usually designed from annual flowering and decorative deciduous plants (with a very limited range) on a lawn background. Ornamental grasses and herbaceous perennials were not commonly used in the urban flower beds.

Modern ecological - naturalistic vision of the park flower beds and ground cover promotes the expansion of the range of herbaceous plants with the involvement of ornamental perennials and grasses close in their image to the plants of natural plant communities as well as the plants of the natural phytocoenosis that can grow together in the same area under the same environmental conditions.

\footnotetext{
${ }^{*}$ Corresponding author: shla-svetlana@yandex.ru
} 
Table 1. The range of plants for greenbelt setting of open spaces (meadow phytocoenosis)

\begin{tabular}{|c|c|c|c|c|c|c|c|c|c|}
\hline \multirow[t]{2}{*}{ № } & \multirow{2}{*}{$\begin{array}{l}\text { Latin } \\
\text { name }\end{array}$} & \multirow[t]{2}{*}{ Family } & \multirow[t]{2}{*}{ Planting type* } & \multirow{2}{*}{$\begin{array}{l}\text { Storey of } \\
\text { planting } * *\end{array}$} & \multicolumn{3}{|c|}{ Ecological conditions } & \multirow{2}{*}{$\begin{array}{c}\text { Flowering } \\
\text { period }\end{array}$} & \multirow[t]{2}{*}{ Notes } \\
\hline & & & & & $\begin{array}{c}\text { Illuminati } \\
\text { on*** }\end{array}$ & $\underset{* *}{\operatorname{Humidity}} * *$ & $\begin{array}{l}\mathrm{pH} \text { and soil } \\
\text { fertility }\end{array}$ & & \\
\hline & $\begin{array}{l}\text { Calamagr } \\
\text { ostis } \\
\text { acutiflora }\end{array}$ & $\begin{array}{c}\text { Grasses } \\
\text { (Poaceae) }\end{array}$ & $\begin{array}{c}\text { Mono } \\
\text { plantings, } \\
\text { matrix, } \\
\text { mixed }\end{array}$ & $\begin{array}{c}\text { Upper, } 150- \\
200 \mathrm{~cm}\end{array}$ & Sun & Drainage & Any soils & VII & $\begin{array}{c}\text { The leaves are } \\
\text { golden - yellow } \\
\text { in autumn. } \\
\text { Aggressive }\end{array}$ \\
\hline & $\begin{array}{c}\text { Phalaris } \\
\text { arundinac } \\
\text { ea }\end{array}$ & $\begin{array}{c}\text { Grasses } \\
\text { (Poaceae) }\end{array}$ & $\begin{array}{c}\text { Mono } \\
\text { plantings, } \\
\text { matrix, } \\
\text { mixed }\end{array}$ & $\begin{array}{c}\text { Upper, } 100- \\
150 \mathrm{~cm}\end{array}$ & Sun & $\begin{array}{l}\text { Excessive } \\
\text { but the } \\
\text { plant is } \\
\text { drought } \\
\text { resistant }\end{array}$ & $\begin{array}{l}\text { Any soils, } \\
\text { pH: } 6.0-8.1\end{array}$ & VI & Aggressive \\
\hline & $\begin{array}{l}\text { Glyceria } \\
\text { maxima }\end{array}$ & $\begin{array}{c}\text { Grasses } \\
\text { (Poaceae) }\end{array}$ & $\begin{array}{c}\text { Mono } \\
\text { plantings, } \\
\text { matrix, } \\
\text { mixed }\end{array}$ & $\begin{array}{l}\text { Medium, 50- } \\
60 \mathrm{~cm}\end{array}$ & $\begin{array}{c}\text { Sun, } \\
\text { semishade }\end{array}$ & Excessive & $\begin{array}{c}\text { Light loam } \\
\text { and peat soils, } \\
\text { pH: } 6.0-6.5\end{array}$ & VI-VIII & $\begin{array}{l}\text { Leaves in } \\
\text { autumn turn a } \\
\text { reddish tone }\end{array}$ \\
\hline & $\begin{array}{c}\text { Miscanthu } \\
\text { s sinensis }\end{array}$ & $\begin{array}{c}\text { Grasses } \\
\text { (Poaceae) }\end{array}$ & $\begin{array}{c}\text { Mono } \\
\text { plantings, } \\
\text { matrix, } \\
\text { mixed, } \\
\text { single }\end{array}$ & $\begin{array}{l}\text { Upper, 20- } \\
250 \mathrm{~cm}\end{array}$ & $\begin{array}{c}\text { Sun, } \\
\text { semishade }\end{array}$ & Sufficient & $\begin{array}{l}\text { Light loam } \\
\text { and peat soil, } \\
\text { pH: } 6.1-7.8\end{array}$ & VII-VIII & $\begin{array}{l}\text { Leaves in } \\
\text { autumn turn } \\
\text { yellow }\end{array}$ \\
\hline & $\begin{array}{c}\text { Deschamp } \\
\text { sia } \\
\text { cespitosa }\end{array}$ & $\begin{array}{c}\text { Grasses } \\
\text { (Poaceae) }\end{array}$ & $\begin{array}{l}\text { Mono } \\
\text { plantings, } \\
\text { matrix, } \\
\text { mixed }\end{array}$ & $\begin{array}{l}\text { Medium and } \\
\text { upper, } \\
30-100 \mathrm{~cm}\end{array}$ & $\begin{array}{c}\text { Sun, } \\
\text { semishade }\end{array}$ & Sufficient & $\begin{array}{l}\text { Any soils, } \\
\text { pH: } 3.7-8.0\end{array}$ & V-VII & - \\
\hline & $\begin{array}{l}\text { Carex } \\
\text { hirta }\end{array}$ & $\begin{array}{l}\text { Sedge family } \\
\text { (Cyperaceae) }\end{array}$ & $\begin{array}{c}\text { Mono } \\
\text { plantings, } \\
\text { matrix, } \\
\text { mixed, } \\
\text { blocks }\end{array}$ & $\begin{array}{l}\text { Bottom and } \\
\text { medium, } \\
10-60 \mathrm{~cm}\end{array}$ & $\begin{array}{c}\text { Sun, } \\
\text { semishade }\end{array}$ & Sufficient & $\begin{array}{l}\text { From wet clay } \\
\text { to dry, sandy }\end{array}$ & IV-VI & Aggressive \\
\hline & $\begin{array}{l}\text { Carex } \\
\text { acuta }\end{array}$ & $\begin{array}{c}\text { Sedge family } \\
- \\
\text { Cyperaceae }\end{array}$ & $\begin{array}{c}\text { Mono plan } \\
\text { tings, matrix, } \\
\text { mixed, } \\
\text { blocks }\end{array}$ & $\begin{array}{c}\text { Medium, 40- } \\
100 \mathrm{~cm}\end{array}$ & $\begin{array}{c}\text { Sun, } \\
\text { semishade }\end{array}$ & $\begin{array}{c}\text { Excessive. } \\
\text { Riparial } \\
\text { plant }\end{array}$ & $\begin{array}{c}\text { Any soils, but } \\
\text { better muddy }\end{array}$ & VI & Aggressive \\
\hline & $\begin{array}{c}\text { Primula } \\
\text { veris }\end{array}$ & $\begin{array}{c}\text { Primrose } \\
\text { (Primulaceae } \\
\text { ) }\end{array}$ & $\begin{array}{l}\text { Mixed, } \\
\text { blocks }\end{array}$ & $\begin{array}{l}\text { Bottom, } 30 \\
\mathrm{~cm}\end{array}$ & Semishade & Sufficient & Light soil & \begin{tabular}{|c|}
$\mathrm{V}-\mathrm{VI}$ \\
Yellow color
\end{tabular} & \\
\hline & $\begin{array}{c}\text { Ranunculu } \\
\text { s ficaria }\end{array}$ & $\begin{array}{c}\text { Crowfoot } \\
\text { family } \\
\text { (Ranunculac } \\
\text { eae) }\end{array}$ & $\begin{array}{l}\text { Mixed, } \\
\text { blocks }\end{array}$ & $\begin{array}{c}\text { Bottom, 5-30 } \\
\mathrm{cm}\end{array}$ & Sun & Sufficient & $\begin{array}{l}\text { Loam, } \\
\text { pH: } 6.5\end{array}$ & \begin{tabular}{|c|} 
IV-V \\
Yellow color
\end{tabular} & $\begin{array}{l}\text { In June, the } \\
\text { aerial part of } \\
\text { the plant dies }\end{array}$ \\
\hline & $\begin{array}{l}\text { Anemone } \\
\text { nemorosa }\end{array}$ & \begin{tabular}{|c} 
Crowfoot \\
family - \\
(Ranunculac \\
eae) \\
\end{tabular} & Mixed, block & $\begin{array}{l}\text { Bottom, } 25 \\
\text { cm }\end{array}$ & $\begin{array}{c}\text { Semishade } \\
\text {, shade }\end{array}$ & Sufficient & $\begin{array}{l}\text { Light loam, } \\
\text { pH: } 6.5\end{array}$ & $\begin{array}{c}\text { IV-V } \\
\text { White color }\end{array}$ & $\begin{array}{c}\text { Ephemeroid - } \\
\text { the leaves are } \\
\text { dying by mid- } \\
\text { summer }\end{array}$ \\
\hline & $\begin{array}{l}\text { Alchemill } \\
\text { a vulgaris }\end{array}$ & \begin{tabular}{|c|} 
Rose family \\
- Rosaceae
\end{tabular} & $\begin{array}{c}\text { Mono } \\
\text { plantings, } \\
\text { mixed, } \\
\text { blocks }\end{array}$ & $\begin{array}{l}\text { Bottom, } 30 \\
\mathrm{~cm}\end{array}$ & $\begin{array}{c}\text { Sun, } \\
\text { semishade }\end{array}$ & Sufficient & $\begin{array}{l}\text { Loam, } \mathrm{pH}: \\
6.0\end{array}$ & $\begin{array}{c}\mathrm{V}-\mathrm{IX} \\
\text { Green and } \\
\text { yellow color }\end{array}$ & $\begin{array}{c}\text { Decorative } \\
\text { effect is } \\
\text { preserved } \\
\text { throughout the } \\
\text { season }\end{array}$ \\
\hline & $\begin{array}{c}\text { Pulmonari } \\
\text { a obscura }\end{array}$ & $\begin{array}{c}\text { Borage } \\
\text { family - } \\
\text { Boraginacea } \\
\text { e }\end{array}$ & Mixed, block & $\begin{array}{l}\text { Bottom, } 30 \\
\mathrm{~cm}\end{array}$ & $\begin{array}{c}\text { Semishade } \\
\text {, shade }\end{array}$ & Sufficient & $\begin{array}{l}\text { Light loam, } \\
\text { pH: 6.1-7.8 }\end{array}$ & \begin{tabular}{|c|} 
IV-V \\
red-lilac-blue \\
color
\end{tabular} & \\
\hline & $\begin{array}{l}\text { Polygonu } \\
\text { m bistorta }\end{array}$ & $\begin{array}{c}\text { Buckwheat } \\
\text { family - } \\
\text { Polygonacea } \\
\text { e } \\
\end{array}$ & $\begin{array}{c}\text { Mono } \\
\text { plantings, } \\
\text { blocks }\end{array}$ & $\begin{array}{l}\text { Upper, } 100 \\
\mathrm{~cm}\end{array}$ & Sun & Exces-sive & $\begin{array}{c}\text { Any soil, but } \\
\text { better fertile, } \\
\text { peaty }\end{array}$ & $\begin{array}{l}\text { V-VII } \\
\text { Pale pink } \\
\text { color }\end{array}$ & \\
\hline & $\begin{array}{c}\text { Thalictru } \\
\mathrm{m} \\
\text { aquilegiifo } \\
\text { lium }\end{array}$ & $\begin{array}{c}\text { Crowfoot } \\
\text { family - } \\
\text { Ranun- } \\
\text { culaceae }\end{array}$ & $\begin{array}{l}\text { Matriz, } \\
\text { mixed }\end{array}$ & $\begin{array}{l}\text { Upper, } 150 \\
\text { cm }\end{array}$ & $\begin{array}{c}\text { Sun, } \\
\text { semishade }\end{array}$ & Sufficient & $\begin{array}{l}\text { Light loam, } \\
\text { pH: } 6.5\end{array}$ & $\begin{array}{c}\text { VI-VII } \\
\text { White, } \\
\text { purple color }\end{array}$ & \\
\hline
\end{tabular}


Table 1. Ending

\begin{tabular}{|c|c|c|c|c|c|c|c|c|}
\hline $\begin{array}{l}\text { Campanul } \\
\text { a latifolia }\end{array}$ & $\begin{array}{c}\text { Bell-flower } \\
\text { family- } \\
\text { Campanulac } \\
\text { eae }\end{array}$ & $\begin{array}{c}\text { Temporary } \\
\text { plantings, } \\
\text { mixed }\end{array}$ & $\begin{array}{l}\text { Upper, } 70- \\
150 \mathrm{~cm}\end{array}$ & $\begin{array}{c}\text { Sun, } \\
\text { semishade }\end{array}$ & Sufficient & $\begin{array}{l}\text { Light loam, } \\
\text { pH: } 6.5-7.0\end{array}$ & $\begin{array}{l}\text { VI-VII } \\
\text { White, } \\
\text { purple, blue } \\
\text { color }\end{array}$ & \\
\hline $\begin{array}{l}\text { Leucanthe } \\
\text { mum } \\
\text { vulgare }\end{array}$ & $\begin{array}{l}\text { Aster family } \\
\text {-Asteraceae }\end{array}$ & $\begin{array}{c}\text { Temporary } \\
\text { plantings, } \\
\text { mixed }\end{array}$ & $\begin{array}{c}\text { Medium, } 45 \\
\mathrm{~cm}\end{array}$ & Sun & Sufficient & Any soils & $\begin{array}{c}\text { VI } \\
\text { White color }\end{array}$ & \\
\hline $\begin{array}{l}\text { Tanacetu } \\
\text { m vulgare }\end{array}$ & $\begin{array}{l}\text { Aster family } \\
\text {-Asteraceae }\end{array}$ & $\begin{array}{c}\text { Temporary } \\
\text { plantings, } \\
\text { mixed }\end{array}$ & $\begin{array}{l}\text { Upper, 80- } \\
120 \mathrm{~cm}\end{array}$ & Sun & Sufficient & Any soils & $\begin{array}{c}\text { VII-X } \\
\text { Yellow color }\end{array}$ & $\begin{array}{l}\text { Undemanding } \\
\text { plant }\end{array}$ \\
\hline $\begin{array}{c}\text { Geranium } \\
\text { pratense }\end{array}$ & $\begin{array}{l}\text { Geranium } \\
\text { family - } \\
\text { Geranium }\end{array}$ & $\begin{array}{c}\text { Temporary } \\
\text { plantings, } \\
\text { mixed }\end{array}$ & $\begin{array}{c}\text { Medium, } 60 \\
\mathrm{~cm}\end{array}$ & Sun & Sufficient & Light loam & $\begin{array}{l}\text { VI-IX Pink } \\
\text { color }\end{array}$ & $\begin{array}{l}\text { Undemanding } \\
\text { plant }\end{array}$ \\
\hline $\begin{array}{l}\text { Lythrum } \\
\text { salicaria }\end{array}$ & $\begin{array}{c}\text { Lythrum } \\
\text { family } \\
\text { Lythraceae } \\
\end{array}$ & $\begin{array}{c}\text { Temporary } \\
\text { plantings, } \\
\text { mixed } \\
\end{array}$ & $\begin{array}{c}\text { Upper, } 80- \\
120 \mathrm{~cm}\end{array}$ & $\begin{array}{c}\text { Sun, } \\
\text { semishade }\end{array}$ & Sufficient & Any soil & $\begin{array}{l}\text { VI-IX } \\
\text { Purplepink } \\
\text { color } \\
\end{array}$ & $\begin{array}{c}\text { Tolerates } \\
\text { stagnant over- } \\
\text { wetting } \\
\end{array}$ \\
\hline $\begin{array}{c}\text { Salvia } \\
\text { pratensis }\end{array}$ & $\begin{array}{l}\text { Deadnettle } \\
\text { family- } \\
\text { Lamiaceae }\end{array}$ & $\begin{array}{c}\text { Temporary } \\
\text { plantings, } \\
\text { mixed, } \\
\text { blocks } \\
\end{array}$ & $\begin{array}{l}\text { Medium, 50- } \\
70 \mathrm{~cm}\end{array}$ & $\begin{array}{c}\text { Sun, } \\
\text { semishade }\end{array}$ & Sufficient & $\begin{array}{l}\text { Any soisl, } \\
\text { pH: } 6.5\end{array}$ & $\begin{array}{c}\text { VI-IX } \\
\text { Pink blue- } \\
\text { violet color }\end{array}$ & $\begin{array}{l}\text { Volatile oil } \\
\text { plant }\end{array}$ \\
\hline $\begin{array}{l}\text { Veronica } \\
\text { spicata }\end{array}$ & $\begin{array}{c}\text { Snapdragon } \\
\text { family - } \\
\text { Scrophu } \\
\text { lariaceae }\end{array}$ & $\begin{array}{l}\text { Temporary } \\
\text { plantings, } \\
\text { mixed, } \\
\text { blocks }\end{array}$ & $\begin{array}{c}\text { Medium, } 70 \\
\mathrm{~cm}\end{array}$ & Sun & Sufficient & $\begin{array}{l}\text { Light loam, } \\
\text { pH: } 6.5-7.0\end{array}$ & $\begin{array}{c}\text { VI } \\
\text { bright blue, } \\
\text { blue color }\end{array}$ & \\
\hline $\begin{array}{l}\text { Verbascu } \\
\mathrm{m} \\
\text { phlomoide } \\
\mathrm{s}\end{array}$ & $\begin{array}{c}\text { Snapdragon } \\
\text { family - } \\
\text { Scrophula } \\
\text { riaceae }\end{array}$ & $\begin{array}{c}\text { Temporary } \\
\text { plantings, } \\
\text { mixed, } \\
\text { blocks }\end{array}$ & $\begin{array}{l}\text { Upper, 80- } \\
200 \mathrm{~cm}\end{array}$ & Sun & Sufficient & Light soils & $\begin{array}{c}\text { VI-X } \\
\text { Yellow color }\end{array}$ & $\begin{array}{c}\text { Biennial plant, } \\
\text { environmentall } \\
\text { y plastic, high } \\
\text { reproduction } \\
\text { rate } \\
\end{array}$ \\
\hline $\begin{array}{l}\text { Nepeta } \times \\
\text { faassenii }\end{array}$ & $\begin{array}{c}\text { Deadnettle } \\
\text { family - } \\
\text { Lamiaceae }\end{array}$ & $\begin{array}{l}\text { Temporary } \\
\text { plantings, } \\
\text { mixed, } \\
\text { blocks }\end{array}$ & $\begin{array}{l}\text { Medium, } 30- \\
60 \mathrm{~cm}\end{array}$ & Sun & Sufficient & $\begin{array}{l}\text { Light loam, } \\
\text { pH: } 6.1-7.8\end{array}$ & $\begin{array}{l}\text { VI-IX } \\
\text { Lavender } \\
\text { color }\end{array}$ & $\begin{array}{l}\text { Pruning of } \\
\text { withered } \\
\text { blossoms to } \\
\text { encourage } \\
\text { reflowering }\end{array}$ \\
\hline $\begin{array}{c}\text { Tulipa } \\
\text { acuminata }\end{array}$ & $\begin{array}{l}\text { Lily family } \\
\text { Liliaceae }\end{array}$ & $\begin{array}{c}\text { Temporary } \\
\text { plantings, } \\
\text { mixed, } \\
\text { blocks }\end{array}$ & $\begin{array}{c}\text { Medium, } 40 \\
\mathrm{~cm}\end{array}$ & Sun & Sufficient & $\begin{array}{l}\text { Sabulous clay } \\
\text { and light loam }\end{array}$ & $\begin{array}{c}\mathrm{V} \\
\text { Yellow with } \\
\text { red stripes }\end{array}$ & $\begin{array}{l}\text { Ephemeroid - } \\
\text { leaves are dying } \\
\text { by mid-summer }\end{array}$ \\
\hline
\end{tabular}

Notes.* Planting type: mono plantings, matrix, temporary plantings, blocks, mixed, single.

** Planting storey: bottom $(10-30 \mathrm{~cm})$, medium $(30-70 \mathrm{~cm})$, upper $(70-140 \mathrm{~cm})$

*** Illumination: shade, semi-shade, sun

***** Humidity: insufficient, sufficient, excessive

Tulipa acuminata, which is not typical for the natural phytocoenosis of the Non-Chernozem zone, was introduced into the proposed range to give more colorfulness to plantings in spring, especially since its appearance does not stand out from the general context.

One of the main tasks of a landscape designer working on the creation of park sceneries is the development of the original exclusive design providing the change of impressions. One of the possibilities to achieve this effect when creating pictures and landscapes in the style of "naturgarten" for different functional areas of the object (parade, walking, quiet recreation area), is to create compositions based on natural or more cultivated and even exquisite image of the same species of plants, but obtained as a result of selection $[1,2]$.

In this article, the criterion for plants selection was their natural appearance and high winter hardiness. Such plants can be used to create a ground cover in the walking area of the park in accordance with the natural light conditions [3].
In the master's dissertation of N.K. Borisova it is shown that currently, to create a sustainable vegetation cover, especially in the urban conditions, the principles of combinatorial planting developed by $\mathrm{P}$. Udolf have been introduced into the practice of the landscape design [4]. In particular, in accordance with the technology of the "New wave", which contributes to the creation of a natural way of planting, the following types of plantings of herbaceous perennial plants were proposed: mono plantings (single and blocks), which consist of one species or plant variety, and combinatorial plantings (blocks, matrix and mixed) that are a collection of the perennial plants of various species that form a stable vegetation cover with a high decorative effect in these specific conditions [5]. 
Table 2. The range of plants for greenbelt setting of closed and semi-open spaces (forest phytocoenosis)

\begin{tabular}{|c|c|c|c|c|c|c|c|c|c|}
\hline \multirow{2}{*}{\begin{tabular}{|l|} 
№ \\
\end{tabular}} & \multirow[t]{2}{*}{ Latin name } & \multirow[t]{2}{*}{ Family } & \multirow[t]{2}{*}{ Planting type } & \multirow{2}{*}{$\begin{array}{c}\text { Storey of } \\
\text { planting } * *\end{array}$} & \multicolumn{3}{|c|}{ Ecological condition } & \multirow{2}{*}{$\begin{array}{c}\text { Flowe-ring } \\
\text { period }\end{array}$} & \multirow[t]{2}{*}{ Notes } \\
\hline & & & & & Illumination $_{* *}^{*}$ & $\underset{* *}{\text { Humidity }}{ }^{* *}$ & $\begin{array}{c}\mathrm{pH} \text { and soil } \\
\text { fertility }\end{array}$ & & \\
\hline 1 & $\begin{array}{l}\text { Molinia } \\
\text { caerulea }\end{array}$ & Poaceae & $\begin{array}{c}\text { Mono } \\
\text { plantings, } \\
\text { matrix, mixed } \\
\end{array}$ & $\begin{array}{l}\text { Medium - } \\
70 \mathrm{~cm}\end{array}$ & $\begin{array}{c}\text { Sun, } \\
\text { semishade }\end{array}$ & Sufficient & $\begin{array}{l}\text { Any soils, } \\
\text { pH: } 6.0-8.0\end{array}$ & VIII-IX & \\
\hline 2 & $\begin{array}{c}\text { Luzula } \\
\text { sylvatica }\end{array}$ & Juncaceae & $\begin{array}{c}\text { Mono } \\
\text { plantings, } \\
\text { matrix, mixed }\end{array}$ & $\begin{array}{l}\text { Bottom, } \\
\text { medium, } \\
20-70 \mathrm{~cm}\end{array}$ & $\begin{array}{l}\text { Semishade, } \\
\text { shade }\end{array}$ & Sufficient & $\begin{array}{l}\text { Light soil, } \\
\text { loam and } \\
\text { peat soil, } \\
\text { pH: } 5.1-9.0\end{array}$ & $\mathrm{~V}-\mathrm{VI}$ & $\begin{array}{c}\text { Grows early } \\
\text { in spring }\end{array}$ \\
\hline 4 & $\begin{array}{c}\text { Sagina } \\
\text { subulata }\end{array}$ & Caryophyllaceae & $\begin{array}{c}\text { Mono } \\
\text { plantings }\end{array}$ & $\begin{array}{l}\text { Bottom } \\
5-10 \mathrm{~cm}\end{array}$ & Sun & Sufficient & $\begin{array}{c}\text { Sandy } \\
\text { loam, } \\
\text { loamy soils }\end{array}$ & VI-IX & $\begin{array}{c}\text { Looks like } \\
\text { moss }\end{array}$ \\
\hline 5 & Poa pratensis & Poaceae & $\begin{array}{c}\text { Mono } \\
\text { plantings, } \\
\text { matrix }\end{array}$ & $\begin{array}{l}\text { Bottom, } \\
20-70 \mathrm{~cm}\end{array}$ & $\begin{array}{c}\text { Sun, } \\
\text { semishade }\end{array}$ & Sufficient & $\begin{array}{l}\text { Any soil } \\
\text { except } \\
\text { boggy }\end{array}$ & VI & \\
\hline 6 & $\begin{array}{l}\text { Milium } \\
\text { effusum }\end{array}$ & Poaceae & Matrix, mixed & $\begin{array}{c}\text { Upper, } 100 \\
\mathrm{~cm}\end{array}$ & $\begin{array}{c}\text { Shade, } \\
\text { semishade }\end{array}$ & Sufficient & $\begin{array}{c}\text { Light soils, } \\
\text { loam, } \\
\text { pH: } 6.5\end{array}$ & VI-VII & \\
\hline 7 & $\begin{array}{l}\text { Corydalis } \\
\text { solida) }\end{array}$ & Fumarioideae & Mixed, blocks & $\begin{array}{l}\text { Bottom, } \\
30 \mathrm{~cm}\end{array}$ & $\begin{array}{l}\text { Sun, semi- } \\
\text { shade }\end{array}$ & Sufficient & \begin{tabular}{|c} 
Light soils, \\
loam, H: \\
$6.1-7.8$ \\
\end{tabular} & $\begin{array}{c}\text { IV-V } \\
\text { Pink color }\end{array}$ & Epheme-roid \\
\hline 8 & $\begin{array}{c}\text { Hepatica } \\
\text { nobilis }\end{array}$ & Ranunculaceae & $\begin{array}{l}\text { "Excipients", } \\
\text { mixed, blocks }\end{array}$ & $\begin{array}{c}\text { Bottom, } \\
15 \mathrm{~cm}\end{array}$ & $\begin{array}{c}\text { Shade, } \\
\text { semishade }\end{array}$ & Sufficient & $\begin{array}{l}\text { Light soils, } \\
\text { loam, } \\
\text { pH: } 7.5-8.0\end{array}$ & $\begin{array}{c}\text { IV } \\
\text { Blue, white, } \\
\text { pink color }\end{array}$ & \\
\hline 9 & $\begin{array}{l}\text { Gera-nium } \\
\text { macrorhizum }\end{array}$ & Geranium & $\begin{array}{c}\text { Mono } \\
\text { plantings, } \\
\text { matrix, mixed } \\
\end{array}$ & $\begin{array}{l}\text { Bottom, } \\
20-25 \mathrm{~cm}\end{array}$ & $\begin{array}{c}\text { Sun, } \\
\text { semishade }\end{array}$ & Sufficient & $\begin{array}{l}\text { Light soils, } \\
\text { pH: } 6.5\end{array}$ & $\begin{array}{c}\text { VI } \\
\begin{array}{c}\text { White, pink } \\
\text { color }\end{array} \\
\end{array}$ & \\
\hline 10 & $\begin{array}{c}\text { Campanula } \\
\text { latifolia }\end{array}$ & Campanulaceae & $\begin{array}{l}\text { Temporary } \\
\text { plantings, } \\
\text { mixed }\end{array}$ & \begin{tabular}{|c|} 
Upper, \\
$70-150 \mathrm{~cm}$
\end{tabular} & $\begin{array}{c}\text { Sun, } \\
\text { semishade }\end{array}$ & Sufficient & $\begin{array}{l}\text { Light loam, } \\
\text { pH: } 6.5-7.0\end{array}$ & $\begin{array}{l}\text { VI-VII } \\
\text { White, } \\
\text { purple, blue } \\
\text { color }\end{array}$ & \\
\hline 12 & $\begin{array}{c}\text { Polygonatum } \\
\text { multiflorum }\end{array}$ & Liliaceae & $\begin{array}{c}\text { Mono } \\
\text { plantings, } \\
\text { mixed } \\
\end{array}$ & $\begin{array}{l}\text { Medium, } \\
\text { 30-90 cm }\end{array}$ & $\begin{array}{c}\text { Shade, } \\
\text { semishade }\end{array}$ & Sufficient & $\begin{array}{c}\text { Light soils, } \\
\text { pH: } 6.5\end{array}$ & $\begin{array}{c}\text { V-VI } \\
\text { White color }\end{array}$ & \\
\hline 14 & Aster alpinus & Asteraceae & $\begin{array}{l}\text { Mono } \\
\text { plantings, } \\
\text { mixed }\end{array}$ & $\begin{array}{c}\text { Bottom, } \\
25-30 \mathrm{~cm}\end{array}$ & $\begin{array}{c}\text { Sun, } \\
\text { semishade }\end{array}$ & Sufficient & $\begin{array}{c}\text { Light soils, } \\
\text { loam }\end{array}$ & $\begin{array}{c}\text { V-VI } \\
\text { White, } \\
\text { purple color }\end{array}$ & $\begin{array}{l}\text { Grows well } \\
\text { in a pine } \\
\text { forest }\end{array}$ \\
\hline 15 & $\begin{array}{l}\text { Dryopteris } \\
\text { filixmas }\end{array}$ & Dryopteridaceae & $\begin{array}{c}\text { Mono } \\
\text { plantings, } \\
\text { mixed } \\
\end{array}$ & $\begin{array}{l}\text { Upper, } \\
120 \mathrm{~cm}\end{array}$ & Shade & Sufficient & $\begin{array}{c}\text { Loam, } \\
\text { pH: } 5.1-6.5\end{array}$ & - & $\begin{array}{c}\text { Grows well } \\
\text { in a spruce } \\
\text { forest }\end{array}$ \\
\hline 16 & $\begin{array}{c}\text { Matteuccia } \\
\text { struthiopteris }\end{array}$ & Woodsiaceae & $\begin{array}{c}\text { Mono } \\
\text { plantings, } \\
\text { mixed }\end{array}$ & $\begin{array}{l}\text { Upper, } \\
150 \mathrm{~cm}\end{array}$ & $\begin{array}{c}\text { Sun, } \\
\text { semishade }\end{array}$ & Sufficient & $\begin{array}{l}\text { Peat soils, } \\
\text { loam, } \\
\text { pH: 5.6-6.0 }\end{array}$ & - & \\
\hline 17 & $\begin{array}{c}\text { Rodgersia } \\
\text { aesculifolia }\end{array}$ & Saxifragaceae & $\begin{array}{c}\text { Mono } \\
\text { plantings, } \\
\text { mixed }\end{array}$ & $\begin{array}{l}\text { Upper, } \\
150 \mathrm{~cm}\end{array}$ & $\begin{array}{c}\text { Shade, } \\
\text { semishade }\end{array}$ & Sufficient & Light soils & $\begin{array}{c}\text { VI } \\
\text { White color }\end{array}$ & $\begin{array}{c}\text { When } \\
\text { blooming } \\
\text { leaves are } \\
\text { purple- } \\
\text { bronze, later } \\
\text { green } \\
\end{array}$ \\
\hline 18 & $\begin{array}{l}\text { Podophyllum } \\
\text { peltatum }\end{array}$ & Berberidaceae & $\begin{array}{c}\text { Mono } \\
\text { plantings, } \\
\text { temporary } \\
\text { plantings, } \\
\text { blocks } \\
\end{array}$ & $\begin{array}{c}\text { Medium, } \\
30-50 \mathrm{~cm}\end{array}$ & $\begin{array}{c}\text { Sun, } \\
\text { semishade }\end{array}$ & Sufficient & $\begin{array}{c}\text { Loam, } \mathrm{pH}: \\
4.0-7.0, \text { but } \\
\text { the optimal } \\
\text { slightly } \\
\text { acidic }\end{array}$ & $\begin{array}{c}\mathrm{V}-\mathrm{VI} \\
\text { White color }\end{array}$ & \\
\hline 19 & $\begin{array}{c}\text { Asarum } \\
\text { europeum }\end{array}$ & Aristolochiaceae & $\begin{array}{c}\text { Mono } \\
\text { plantings, } \\
\text { mixed }\end{array}$ & $\begin{array}{l}\text { Bottom, } \\
30 \mathrm{~cm}\end{array}$ & $\begin{array}{c}\text { Shade, } \\
\text { semishade }\end{array}$ & Sufficient & $\begin{array}{l}\text { Light soils, } \\
\text { pH: } 6.5-7.5\end{array}$ & $\begin{array}{l}\text { Flowering a } \\
\text { little notice- } \\
\text { able }\end{array}$ & $\begin{array}{c}\text { Winter-green } \\
\text { plant }\end{array}$ \\
\hline
\end{tabular}

Notes:

* Planting type: mono plantings, matrix, temporary plantings, blocks, mixed, single plantings.

** Planting storey: bottom $(10-30 \mathrm{~cm})$, medium $(30-70 \mathrm{~cm})$, upper $(70-140 \mathrm{~cm})$

*** Illumination: shade, semi-shade, sun

$* * * *$ Humidity: insufficient, sufficient, excessive 
In our research, we selected and analyzed the species of the plants of the natural phytocoenosis that could be used to create a ground park cover along the walking route passing through the open, semi-open and closed park spaces. In each case, there is a specific environmental situation in terms of the illumination of the areas, humidity, fertility and acidity of the soil. In addition, we took into account the seasonal changes in the decorativeness of the plants in order to ensure the aesthetic appeal of the surroundings of the route from spring to autumn.

Most often in the Non-Chernozem zone in the open areas, a meadow plant community is formed, which is dominated by perennial herbaceous plants (sedge and grass families), growing in conditions of sufficient or excessive moisture. A soddy type of soil formation is characteristic for meadows. Depending on the conditions of a particular place, the fertility and moisture content of these soils can be quite different.

The list of the plants for decoration of open areas that contribute to the image of meadow phytocoenosis is presented in Table 1 with the indication of their main environmental requirements, decorative period and planting place.

The forest phytocoenosis, which is developed within semi-open and closed park spaces, is a complex plant community consisting of woody, shrubby vegetation and a ground cover.

In comparison with the closed park spaces, semiopen spaces are lit better due to the sunrays penetrating them.

A characteristic feature of the forest phytocenoses is the formation of the forest litter from the leaf or coniferous litter, in which the root system of herbaceous perennials is located.

The selection of perennial herbaceous plants for the ground cover is recommended in accordance with the species composition of trees in the park.
Taking into account all above stated, the range of perennial grassy plants offered for the design of a ground cover of the closed and semi-open park spaces solved in the style of natural phytocenoses (1) is presented in Table 2 .

In order to achieve the naturalism, the boundaries of artificially created plant communities should be smoothed. This goal may well be realistically, as most of the selected species of the plants have a pronounced plasticity relative to the environmental conditions of the area.

\section{Conclusion}

The proposed range of the herbaceous perennial plants can be used in the design of structural modules of the ground cover for park areas with different environmental conditions.

\section{References}

1. I.Yu. Bochkova, On the use of perennial flower crops in urban landscaping, Questions of landscape architecture, 69, 24 (FGBOU VPO MGUL, 2014)

2. R.A. Karpisonova, Principles of selection of decorative perennials for urban flower beds, GBS Bull., 197, 132-138 (2011)

3. A.V. Smirnov, Atlas of plants of the Moscow region, Retrieved from: http://floralib.msk.ru/index.html.

4. N.K. Borisova, Questions of landscape architecture, in Collection of articles on the mater. of the faculty conf. "Expansion of the practice of application of perennial herbaceous plants in the urban environment", 378, 93 (2015)

5. T. Rainer, K. Vest, Plantings in the post-natural world, 272 (2018) 